\title{
Optical Data Downlinks from Earth Observation Platforms
}

\author{
D. Giggenbach*, J. Horwath, and M. Knapek \\ Institute of Communications and Navigation, German Aerospace Center (DLR), D-82234 Wessling
}

\begin{abstract}
The increasing resolution of earth observation sensors will require much higher data rates for the data downlink in future than is feasible with conventional RF-technology. This applies for earth observation satellites as well as for aeronautic observation platforms, such as aircraft or stratospheric high altitude platforms. The most promising solution for this data downlink bottleneck is the application of optical free space transmission technologies. DLR has built diverse atmospheric flight terminals and performed several trials of optical downlinks from space (together with partnering organizations) as well as from atmospheric carriers in recent years. Here we present and compare results of such communication system trials.
\end{abstract}

Keywords: optical downlink, earth observation platform, low earth orbit satellites, aeronautic platform, real-time observation data

\section{INTRODUCTION}

DLR has performed several trials in the application field of data downlinks for earth-observation (EO) sensors, both from space-based and atmospheric platforms. These namely are the following projects:

A) KIODO (Kirari Optical Downlinks to Oberpfaffenhofen), which performed IM/DD downlinks from the Japanese LEO-satellite OICETS (launched and operated by JAXA) to DLR's optical ground station at Oberpfaffenhofen in June 2006 [1].

B) LCTSX-dIOP (Laser Communications Terminal on TerraSAR-X - downlinks to Oberpfaffenhofen) with signal acquisition, mutual terminal tracking and signal quality assessment from the partner terminal onboard the German SAR-satellite TerraSAR-X. LCT is an optical inter-satellite link terminal with coherent homodyne BPSK transmission format at 1064nm wavelength, build by Tesat-Spacecom under DLR-contract. During these downlink trials no coherent signal reception is performed, but received power and wavefront-quality is measured and the required performance of an appropriate adaptive optics system (AO) is assessed. These experiments are ongoing in 2008 and 2009. The trials are supported by further downlinks to our partners at NICT, Tokyo, and to ESA's optical ground station at Izana, Tenerife.

C) CAPANINA-Trial2: Optical IM/DD downlinks from a stratospheric balloon payload in August 2005 at ESRANGE, Kiruna, Sweden. Platform altitude was up to $24.7 \mathrm{~km}$ and link distance up to $64 \mathrm{~km}$ with variable datarates of $1.25 \mathrm{Gbps}$ and $622 \mathrm{Mbps}[2]$.

D) ARGOS: Optical IM/DD downlinks from an aircraft with Fast-Ethernet data format and distances up to $100 \mathrm{~km}$ [3]. The targeted application scenarios of ARGOS are real-time monitoring of traffic and mass-events. Downlink trials were performed in 2008 with test-data while in 2009 live high-resolution imaging data will be transmitted to ground.

The ground terminal in cases A, B, and D is DLR's Optical Ground Station at Oberpfaffenhofen (OGS-OP), while in case $\mathrm{C}$ a transportable OGS version was brought to and installed at ESRANGE near Kiruna, Sweden. The acquisition procedure in the satellite downlinks was based on open-loop pointing with orbit prediction, while the atmospheric platform links were supported by a GPS-based RF signaling link. After the acquisition of the partner terminal's signal, a tracking signal was generated by optical sensors and mutual optical tracking was performed in all link cases.

*dirk.giggenbach@ dlr.de; www.dlr.de

Copyright 2009 Society of Photo-Optical Instrumentation Engineers. This paper was published in SPIE Free-Space Laser Communications Technologies XXI and is made available as an electronic reprint with permission of SPIE. One print or electronic copy may be made for personal use only. Systematic or multiple reproduction, distribution to multiple locations via electronic or other means, duplication of any material in this paper for a fee or for commercial purposes, or modification of the content of the paper are prohibited. 
Measurements of the received signal in terms of statistics of the Rx-intensity and -power were performed during all trials. This allowed for characterizing the atmospheric optical transmission channel. BER-measurements were performed in cases $\mathrm{A}, \mathrm{C}$, and $\mathrm{D}$.

All experiments have an atmospheric path section in common. This path causes distortions of the optical signal wavefront by atmospheric index-of-refraction turbulence (IRT), an effect that produces fades of the received signal. Another source for fading in all cases are miss-pointing and miss-tracking. Clouds also can cause fading or transient link blockages in cases $\mathrm{A}$ to $\mathrm{C}$ while the aircraft platform usually operates below cloud layers. Compact cloud layers can even totally prevent the optical downlink in cases $\mathrm{A}$ to $\mathrm{C}$, a situation that has to be overcome either by ground station diversity [4] or through a lower-rate RF backup link.

While EO-satellites can provide global coverage, they have infrequent contacts to their ground stations and thus usually only provide stored EO-data, with a delay of minutes to hours depending on geographical situation between sat-orbit, ground stations, and area of interest. Atmospheric platforms on the other hand have limited field of regard and operative range, but with their direct downlinks they can provide real-time observation data at distances up to several hundred kilometers.

In this paper we present and compare the different link constraints and - partly preliminary - trial results.

\section{APPLICATION SCENARIOS FOR OPTICAL DOWNLINKS FROM EARTH OBSERVATION PLATFORMS AND CONSTRAINTS}

\subsection{Low Earth Orbit Platforms}

Earth-observation Satellites in polar low earth orbit (LEO) allow global sensor coverage. One drawback is their limited visibility to one ground station. This usually prevents real-time data access and requires large onboard storage of EOdata. Ground station (GS) locations near the earth's poles allow frequent contacts but operations of these GSs is aggravated. However, sensor operational time is mostly limited by the downlink capacity [4]. Therefore, higher downlink data rates directly allow increased sensor usage. With optical downlinks, the data rate can be boosted from currently 300Mbps X-Band rates to several Gbps.

Not treated here are optical downlinks from geostationary observation satellites as well as GEO-relay scenarios (GEO satellites that relay the data from EO-LEOs down to earth).

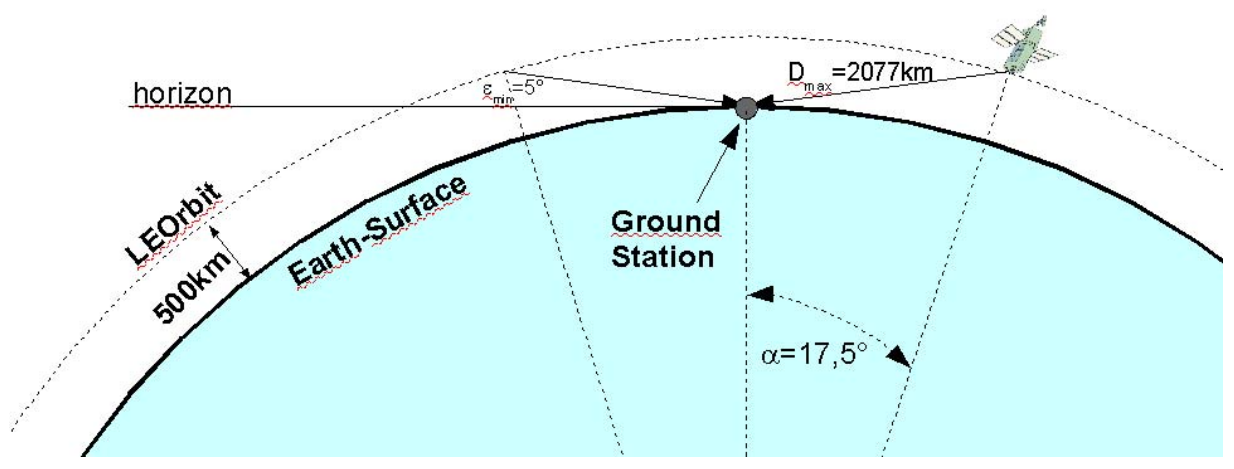

Figure 1: Link geometry of EO-Sat downlink with exemplary values (minimum elevation angle is $5^{\circ}$, ground station at sea level), the maximum time fraction (with zenith-overflight) of one orbit's contact to the ground station is $9,7 \%$, the duration of one downlink is max. 9 minutes (from $5^{\circ}$ to zenith to $5^{\circ}$ elevation). 


\subsection{Stratospheric Platforms}

High-Altitude (stratospheric) Platforms (HAPs) enable several new military, security, as well as commercial applications and they can be used for multiple purposes in parallel. E.g. a HAP could carry a telecom payload for mobile end-user connectivity and monitor agriculture, forest fires, and boarders at the same time [5]. HAPs could even function as relay stations above the clouds for optical downlinks from EO-satellites [4]. Real-time data access is mostly possible and wanted with these kinds of systems. While data could be uplinked to a GEO communications relay satellite, this link provides only low data rate at long delay, generates high costs, and requires high power and mass for the data terminal on the HAP. An optical downlink on the other hand can be blocked by clouds in the line-of-sight towards the ground station, a situation that must be met by RF-backup or ground-station diversity. When a cloud-free line-of-sight is available, the optical link offers multi-Gigabit data rates with minimum mass and power constraints. The online data access link calls for certain quality-of-service requirements different from those of satellite-downlinks, e.g. short delay, fast FEC to provide error-free realtime data, and the ability to directly access the observation data without postprocessing.

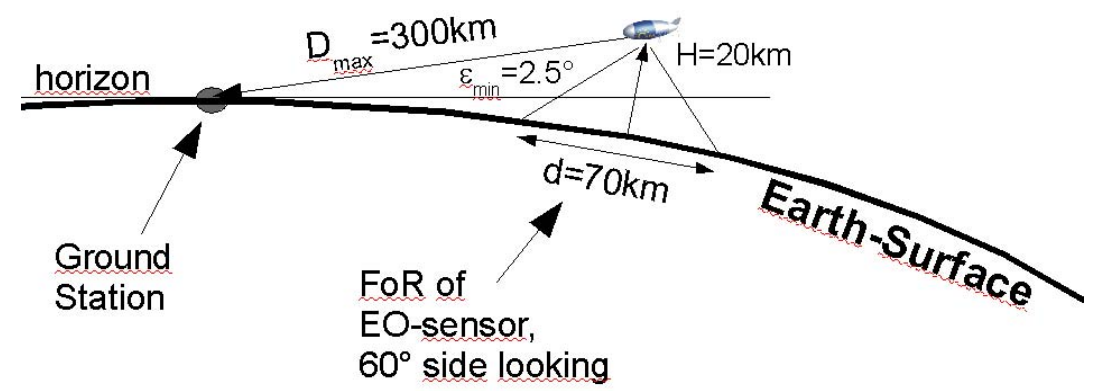

Figure 2: HAP downlink geometry

\subsection{Tropospheric Aircraft Platform:}

Small low-altitude platforms can be deployed very quickly on demand, e.g. as in emergency rescue situations. They can be aerodynamic (fixed- or rotary-wing) as well aerostatic (lighter than air platforms, blimp-like). Their reduced field-ofregard is rather suited for observing objects or events with known location (aircraft can reach target areas very fast) than for general observation purposes. Their low to medium flight altitude on the other hand allows very high resolution images or video-surveillance down to detection and tracking of individual vehicles or persons.

DLR is developing an airborne wide area monitoring system for observation of large-scale events, traffic, and natural disasters. The used data link systems are optical- and microwave links [3]. The observation sensors are based on a high resolution area sensor daylight camera and on a multi-channel full polarimetric SAR system. The advantage of this system is that both, the aircraft and the receiving ground station are relatively fast deployed and can supply the operational headquarter during a disaster to guarantee the situation awareness. Figure 3 shows the downlink geometry of this scenario. 


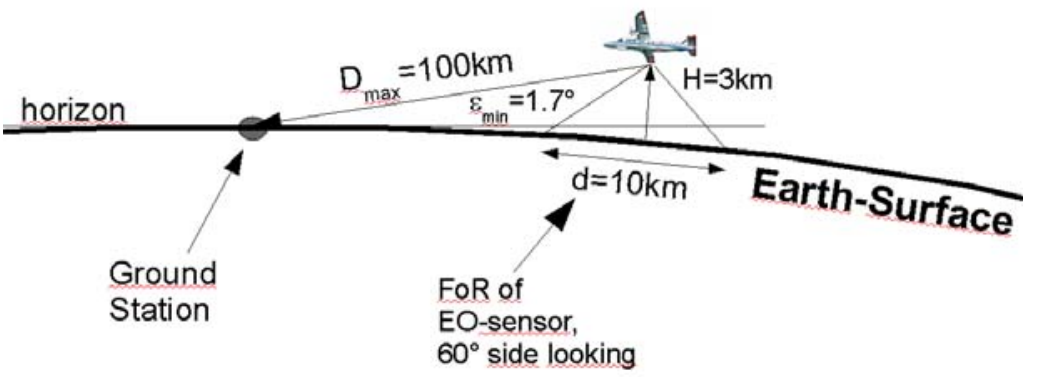

Figure 3: Optical aircraft downlink geometry

\subsection{Link-Blockage by Clouds and its Mitigation}

The optical link can be blocked by thick clouds. For reliable space-downlinks, a network of optical ground stations located several hundred kilometers apart is required to enable ground station diversity. Simulations based on cloud statistics show that long-term system availabilities over $80 \%$ can be reached with no more than four ground stations for Central Europe.

HAP-Downlinks have similar cloud-impairments, but due to their smaller link radius of typical 300km they can not benefit from ground station diversity as much as LEO-downlinks. They rather would need interconnections to other HAPs or aircraft, relaying their data to a cloud-free ground station. Backup links like RF-downlinks or satellite-links would be required in geographical areas with high cloud probability. In deployment areas with low cloud probability (e.g. the Mediterranean or North-Africa) on the other hand, over $90 \%$ can be reached with one or two ground stations.

\subsection{Influence of Atmospheric Index-of-Refraction Turbulence on Link Performance}

The atmospheric index-of-refraction turbulence (IRT) causes the well-known effects of intensity scintillation and wavefront distortions. The high angular slew rate in mobile link scenarios broadens the time-spectra of these effects [1]. Also, the low link elevations lead to long atmospheric path fractions, e.g. several hundred kilometers in LEO satellite downlinks. Therefore, optical downlinks typically are subject to strong turbulence or even saturation.
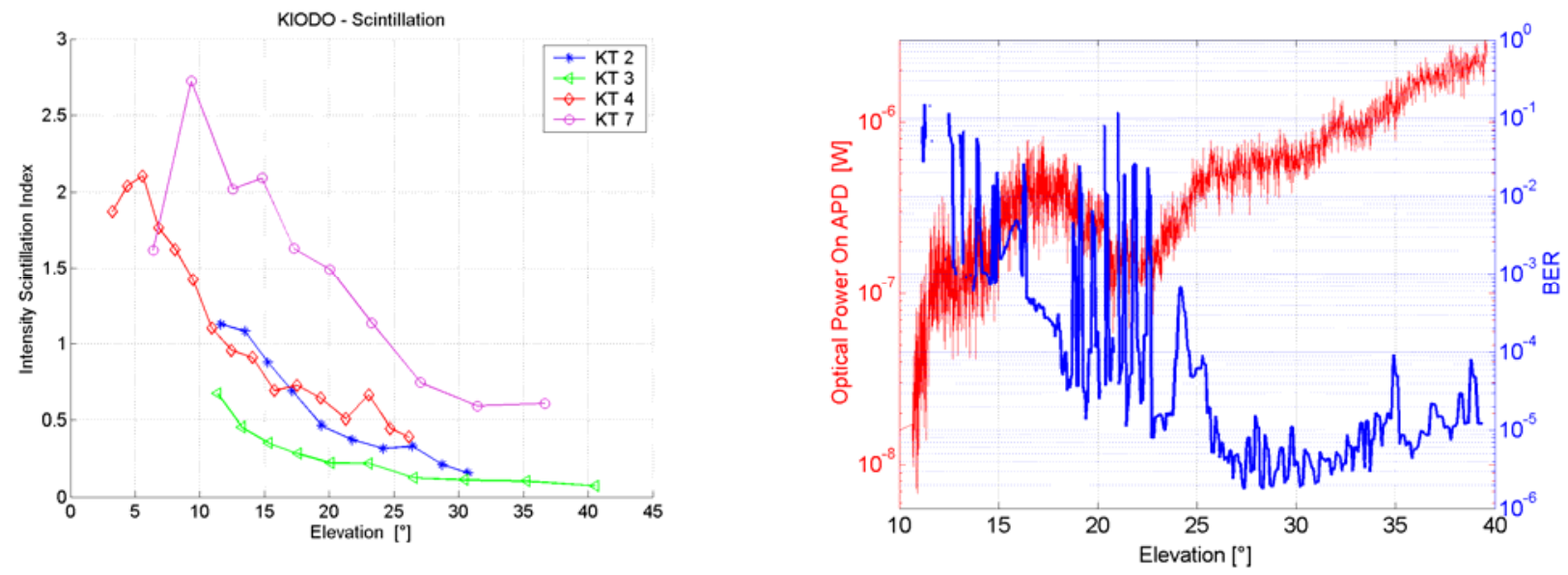

Figure 4: Examples of optical scintillation effects caused by IRT: Measured KIODO intensity scintillation index ${\sigma_{I}^{2}}^{2}$ over elevation at four different trials. One can observe scintillation saturation at low elevations and peaking between $5^{\circ}$ and $10^{\circ}$ (left). Received power and BER of KIODO trial \#7 (right). The relatively high BER was caused by strong fading while the mean received power was more than sufficient. Clouds between $18^{\circ}$ and $25^{\circ}$ elevation caused power degradation and thus BER-surges, while the tracking lock was maintained. 


\subsection{Wavelength Selection}

Besides the question of component availability at different wavelengths, eye-safety is a critical issue for the ground station operators and when the FTs operate near-earth ( $\rightarrow$ aircraft downlinks). 1550nm has the advantage of being approx. a factor of 100 more eye-safe than wavelengths around $800 \mathrm{~nm}$. This allows higher OGS beacon powers. Also in favor of $15 \times x n m$ speaks the reduced background light from celestial bodies, clouds, and earth albedo. This reduces blinding of tracking sensors and thus allows sensors with wider field-of-view. Atmospheric attenuation is very low at $1550 \mathrm{~nm}$, while aerosol scattering remains an issue for all wavelengths below $10 \mu \mathrm{m}$.

\section{VERIFICATION TRIALS OF OPTICAL DOWNLINKS}

\subsection{Description of the Optical Ground Station Oberpfaffenhofen (OGS-OP)}

The DLR optical ground station at Oberpfaffenhofen (OGS-OP) including the atmospheric measurement instruments has been developed under an ESA contract and DLR internal support since 2004 [6]. It has been designed to operate in various scenarios as satellite-to-ground links, links to airplanes and links to stratospheric platforms. The main goal of the OGS-OP is the measurement of atmospheric conditions in parallel to communication performance (i.e. bit-error rate). Basis of the station is a $40 \mathrm{~cm}$ Cassegrain telescope and a custom-made fork-mount. The measurement instruments (Figure 5 right) include devices for wavefront and intensity measurements using the beam from the counter-terminal. Table 1 gives a list of the measurement instruments with the used sensor and the expected results. The functional system architecture is depicted in Figure 6.
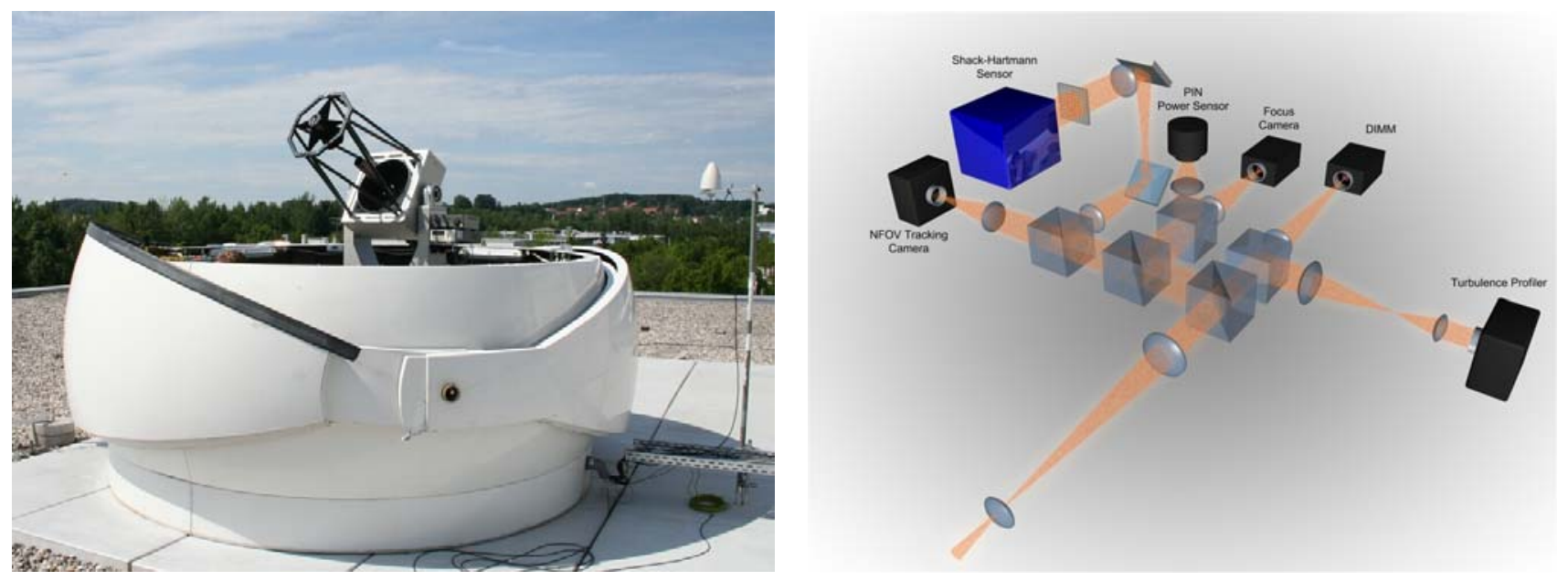

Figure 5: Optical Ground Station Oberpfaffenhofen (OGS-OP). Photograph of dome, mount, and telescope (left), optical path of the measurement devices (right).

OGS-OP - which was used for all trials - allows different tracking modes (optical, orbit, GPS) and operation at several wavelengths. It contains different beacon laser sources $(808 \mathrm{~nm}, 1064 \mathrm{~nm}, 1550 \mathrm{~nm}, 1590 \mathrm{~nm})$ and a range of high sensitive APD receiver frontends ( $\mathrm{Si}$ and $\mathrm{InGaAs}$ for data rates between $50 \mathrm{Mbps}$ and $1.5 \mathrm{Gbps}$ ).

Transmitter diversity is applied as a general means to reduce scintillation at the communications partner. Two co-aligned incoherent beacon sources with a lateral distance of approx. $70 \mathrm{~cm}$ are employed to produce two fairly uncorrelated intensity patterns. 


\begin{tabular}{|l|l|l|}
\hline Instrument & Sensor & Measured Values and Results \\
\hline Turbulence Profiler & Basler A102f $(1392 \times 1040,6.45 \mathrm{um})$ & $\begin{array}{l}\text { High-resolution intensity distribution in the telescope aperture, } \\
\mathrm{C}_{\mathrm{n}}^{2} \text { profile over the beam path, scintillation index } \sigma_{\mathrm{i}} \text {, intensity } \\
\text { correlation length } \rho_{\mathrm{i}} \text {. }\end{array}$ \\
\hline $\begin{array}{l}\text { Dual Image Motion } \\
\text { Monitor }\end{array}$ & $\begin{array}{l}\text { Basler A602f } \\
(656 \mathrm{x} 491,9.9 \mu \mathrm{m}, 100 \mathrm{fps})\end{array}$ & Fried Parameter $\mathrm{r}_{0}$ \\
\hline $\begin{array}{l}\text { Power Sensor (external } \\
5 \mathrm{~cm} \text { aperture) }\end{array}$ & $\begin{array}{l}\text { PIN-Diode Thorlabs PDA400, } \\
3.6 \mathrm{x} 3.6 \mathrm{~mm}, \text { InGaAs, 20ksamples/s }\end{array}$ & Received power with high sampling rate \\
\hline $\begin{array}{l}\text { Power Sensor }(40 \mathrm{~cm} \\
\text { telescope aperture })\end{array}$ & $\begin{array}{l}\text { PIN-Diode Thorlabs PDA55, } \\
3.6 \mathrm{x} 3.6 \mathrm{~mm}, \text { Silicon, 20ksamples/s }\end{array}$ & Received power with high sampling rate \\
\hline Focus Camera & Basler A622f $(1280 \mathrm{x} 1024,6.7 \mathrm{um})$ & Focus intensity distribution, $\mathrm{r}_{0}$, speckle pattern analysis \\
\hline Shack-Hartmann Sensor & $\begin{array}{l}\text { Xenics Cheetah }(640 x 512 \text { Pixels, } \\
20 u m), \text { InGaAs Sensor }\end{array}$ & High-resolution wavefront phase with more than 500fps \\
\hline Tracking Camera & Basler A602f & Tracking capabilities \\
\hline
\end{tabular}

Table 1: Measurement instruments on the DLR optical ground station OGS-OP.

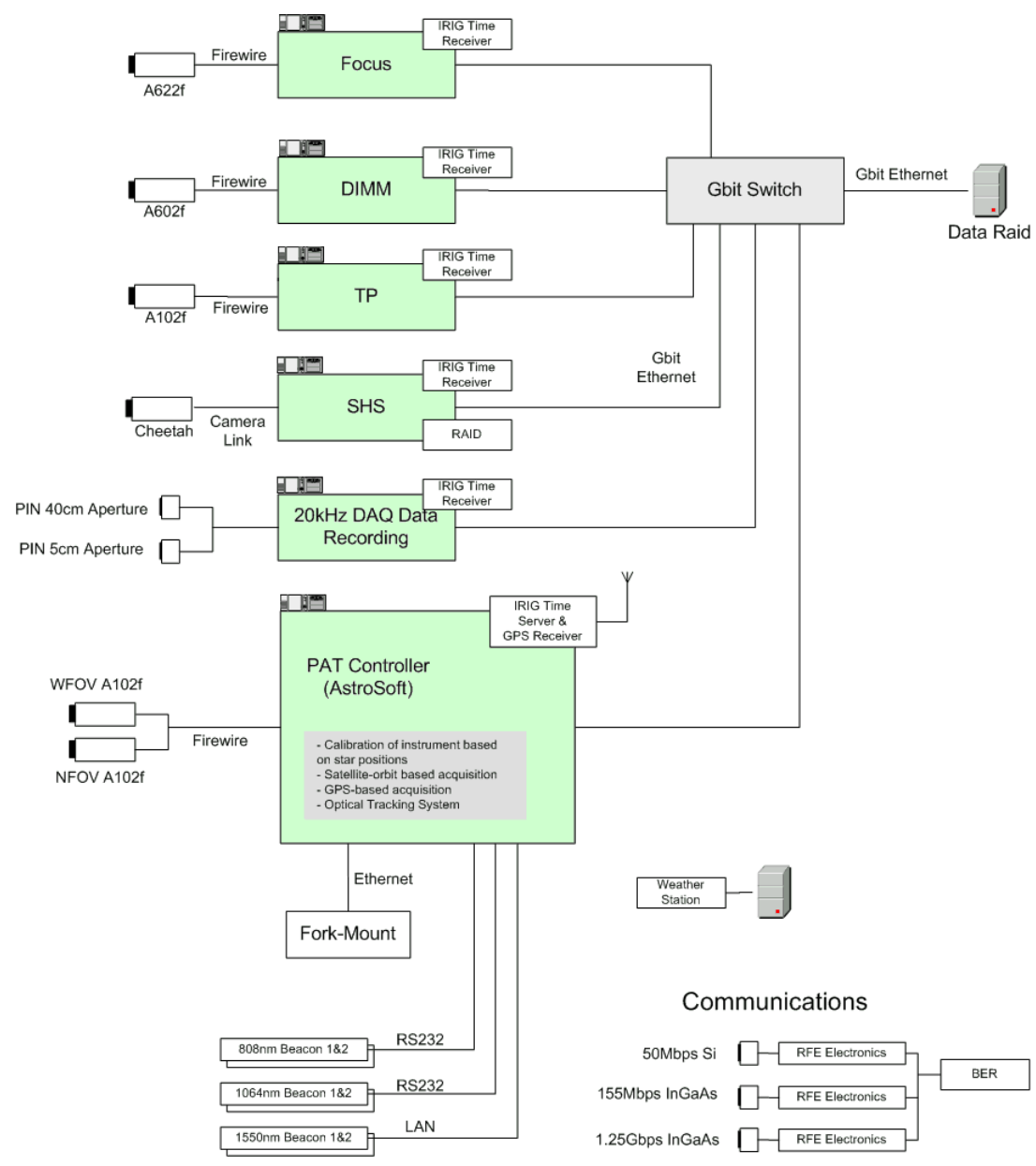

Figure 6: Functional Diagram of the OGS-OP - Atmospheric measurement instruments, PAT system, and communication systems. 


\subsection{IM/DD Satellite-Downlinks: KIODO}

Eight KIODO trials were scheduled during June 2006 of which five were performed successfully. Two of the remaining were hindered by clouds and the third was prevented by a software malfunction of the optical ground station.

BER as low as $2 * 10^{-6}$ could be observed. The remaining error-floor was due to saturation of the APD receiver and slow clock resynchronization after deep fades with loss-of-signal. We believe that error-free reception would have been possible with optimized receiver electronics and using a fast variable optical attenuator (VOA) in front of the APD.

We observed a general agreement of measurements with scintillation theory. Measurements at low elevations have enabled the observation of scintillation saturation. A complete summary of all trial results can be found in [1].

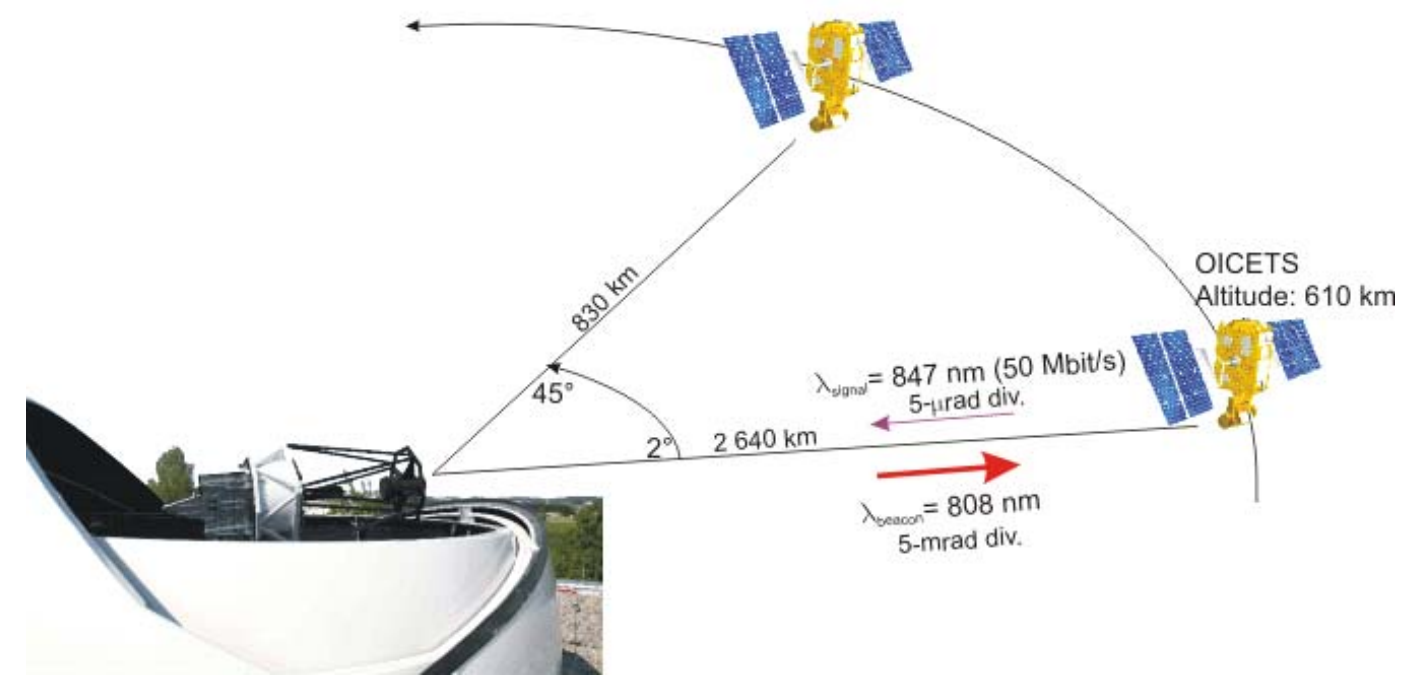

Figure 7: Overview of the KIODO downlink scenario

\subsection{Coherent Satellite-Downlinks: LCTSX-dIOP}

General information on the LCT Inter Satellite Link (ISL) technology and its ISL acquisition procedure can be found in [7]. When using this technology for downlinks, the diffraction limited acquisition scan process of the space terminal is hindered by the atmospheric fading and beam wander. As no coherent Tx-source for the data-uplink is available to us, at OGS-OP we use two divergent incoherent high-power beacons to enable an alternative acquisition and tracking method with the terminal's direct detection fine-acquisition sensors. These can also serve as tracking sensors under certain limitations. No coherent data reception was performed during these trials.

Heterodyning receivers require matching of incoming signal field with the local oscillator. As the atmospheric IRT reduces the signal coherence length, the maximum applicable Rx-aperture is limited to sizes depending on wavelength, elevation, and specific atmospheric IRT-profile. In [8] it is shown that the aperture diameter shall not exceed $2 * \mathrm{r}_{0}$ with angle-of-arrival tracking of the received signal. When the link budget demands larger Rx-apertures, techniques for wavefront correction must be employed, namely adaptive optics (AO). To design an according AO-system, ShackHartmann wavefront measurements were performed during LCTSX-downlinks. 

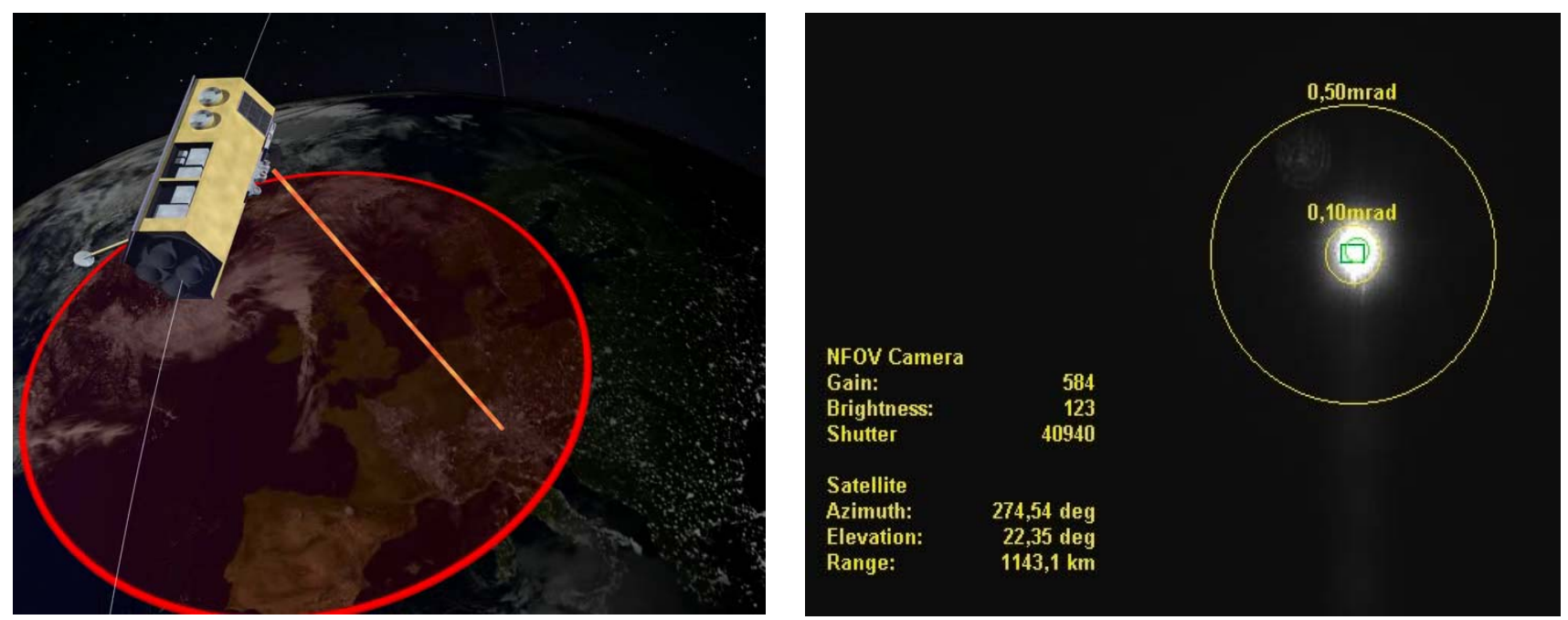

Figure 8: Scenario of LCTSX-Downlink from TerraSAR-X (left), received signal on tracking camera of OGS-OP (right)
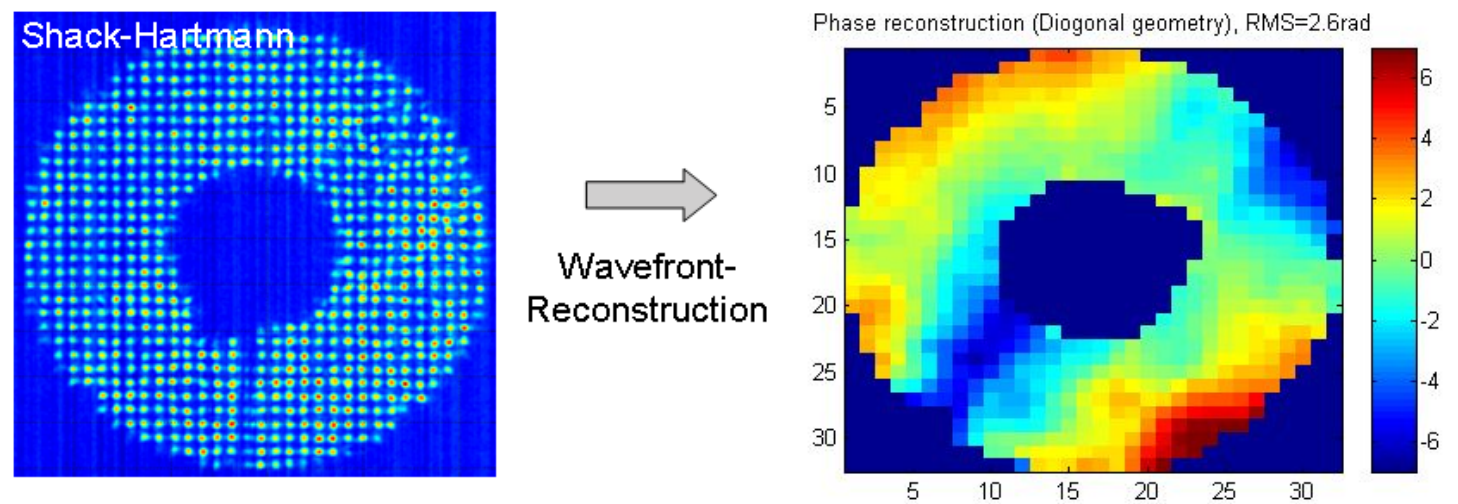

Figure 9: Typical image of the InGaAs Shack-Hartmann sensor with the reconstructed phase for a 400m link between two buildings. Both images show the central obscuration of the OGS-OP $40 \mathrm{~cm}$ Cassegrain telescope.

\subsection{Stratospheric Downlinks from High-Altitude-Platforms: CAPANINA-Trial2}

Within the CAPANINA project, funded by the European Commission under the $6^{\text {th }}$ framework program, the stratospheric optical payload experiment (STROPEX) has been performed. An optical terminal was carried by a 12000 $\mathrm{m}^{3}$ balloon type high altitude test-bed. 70 minutes after launch, the balloon reached the targeted altitude of $23 \mathrm{~km}$ and laser beacons of the ground station were switched on. The optical terminal on the balloon received initial nadir angle information from the ground station control computer in order to shorten the scan time during acquisition. With a selected scan speed of $10 \%$ s the acquisition process lasted 20 seconds. Then the terminal-beacons $(976 \mathrm{~nm})$ were switched on and illuminated the ground station. After acquiring this beacon, the ground station changed from GPS tracking to the closed loop optical tracking and kept the terminal beacon within the field of view (FOV) of the ground station's communication receiver, which was $100 \mu \mathrm{rad}$. Finally, the $1550 \mathrm{~nm}$ communication system of the terminal was activated via the telemetry link and then 8 hours of data transmission tests were performed. 
The high signal to noise ratio enabled a long-term bit error rate (BER) of less than $10^{-9}$ at a bit rate of $622 \mathrm{Mbit} / \mathrm{s}$. Frequently, the BER went down to $10^{-10}$ for several minutes. This high quality signal was received up to a maximum distance of $64.15 \mathrm{~km}$ from the ground station (Figure 10; upper right). The $1.25 \mathrm{Gbit} / \mathrm{s}$ transmission was also successful, this eye pattern at maximum distance can be seen in Figure 10 lower right.
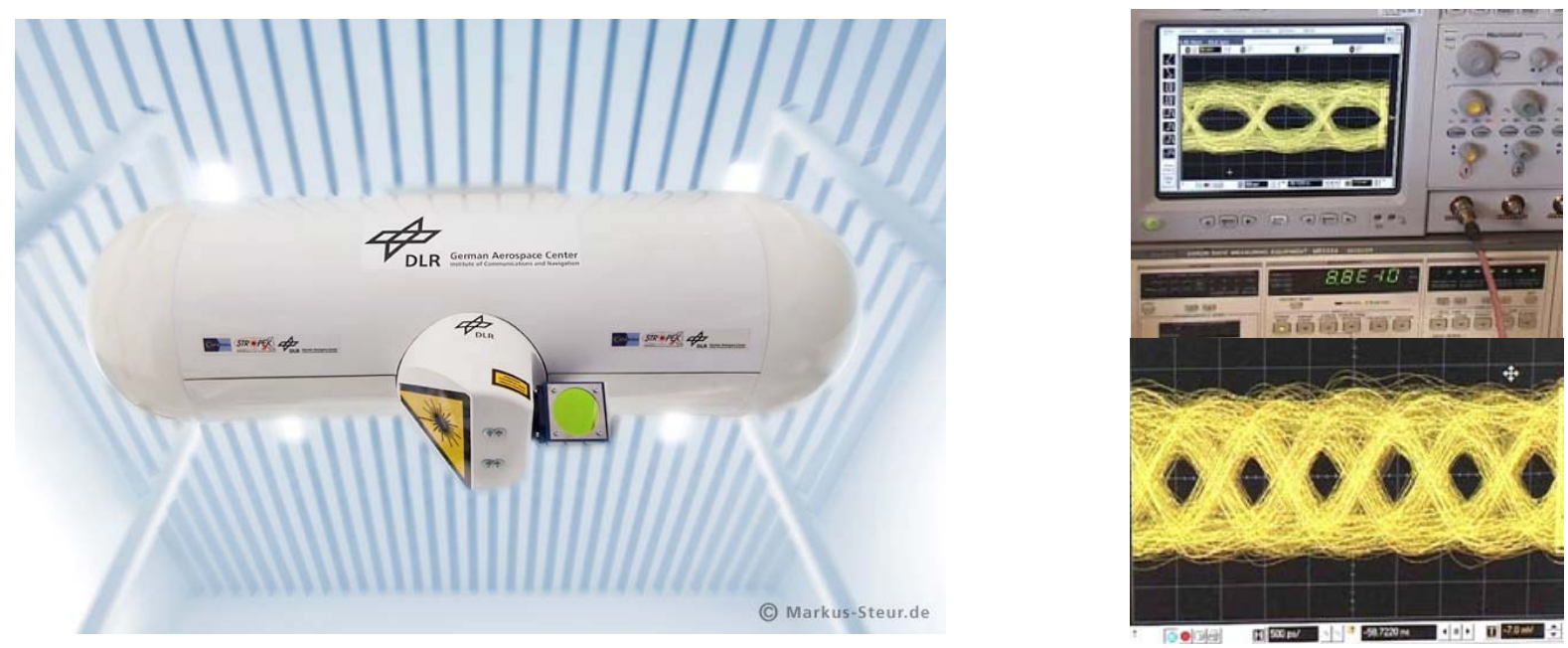

Figure 10: The CAPANINA stratospheric terminal wingpod (left) and the received data eye patterns at the ground station (right). The upper picture shows a data rate of $622 \mathrm{Mbit} / \mathrm{s}$, the lower picture shows $1.25 \mathrm{Mbit} / \mathrm{s}$

\subsection{Aeronautical Downlink from Aircraft: ARGOS}

The DLR-project ARGOS focuses on traffic monitoring and surveillance of mass-events. Sensors are optical (highresolution still camera with up to 48 Mpixel of resolution and 3 frames per second, producing up to 100 Mbps of dataflow) and SAR. An optical downlink custom build by DLR will provide real-time sensor downlink capability at up to $125 \mathrm{Mbps}$ channel rate (Fast-Ethernet). In a next step the data rate shall be increased to $1.25 \mathrm{Gbps}$. As clouds and fog prevent both the operation of the optical sensor as well as the optical downlink, the combination of both technologies is advantageous. For the SAR-sensor, an additional lower data rate RF downlink can be used.

By adopting transparent standard Ethernet format for the optical link, simple interfacing to PC-based sensors and data processing is assured. As packet-based FEC proofed beneficial for the atmospheric IRT fading channel, the Ethernet packet itself is coded (packet-layer FEC), providing the necessary long code words and simple interleaving over the slow fades. Onboard FEC-processing of the sensor data is based on compact-PCI-hardware

Results of first ARGOS-test flights:

Between Nov. 24 and Dec. 42008 several test flights were executed with DLR's Do228 aircraft. GPS-assisted acquisition and mutual optical tracking of FT and OGS-OP could be performed at up to $85 \mathrm{~km}$. Data communication tests with PRBS-data have been performed up to $40 \mathrm{~km}$ with short-term BERs of below $10^{-8}$. An in-depth description of the experiments can be found in [3].

A schematic of the terminal and ground station setup can be seen in Figure 12. 

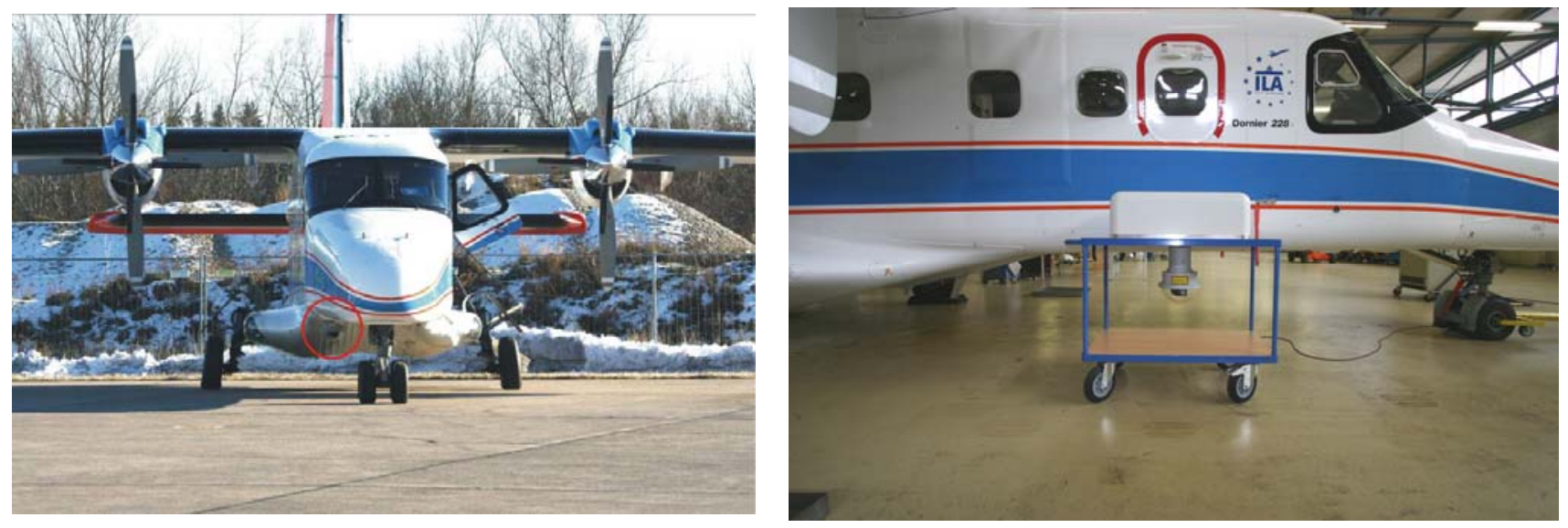

Figure 11: Photograph of the ARGOS-terminal mounted underneath the Do-228 research-aircraft (left) and terminal prior to integration (right).

Optical Aircraft Terminal

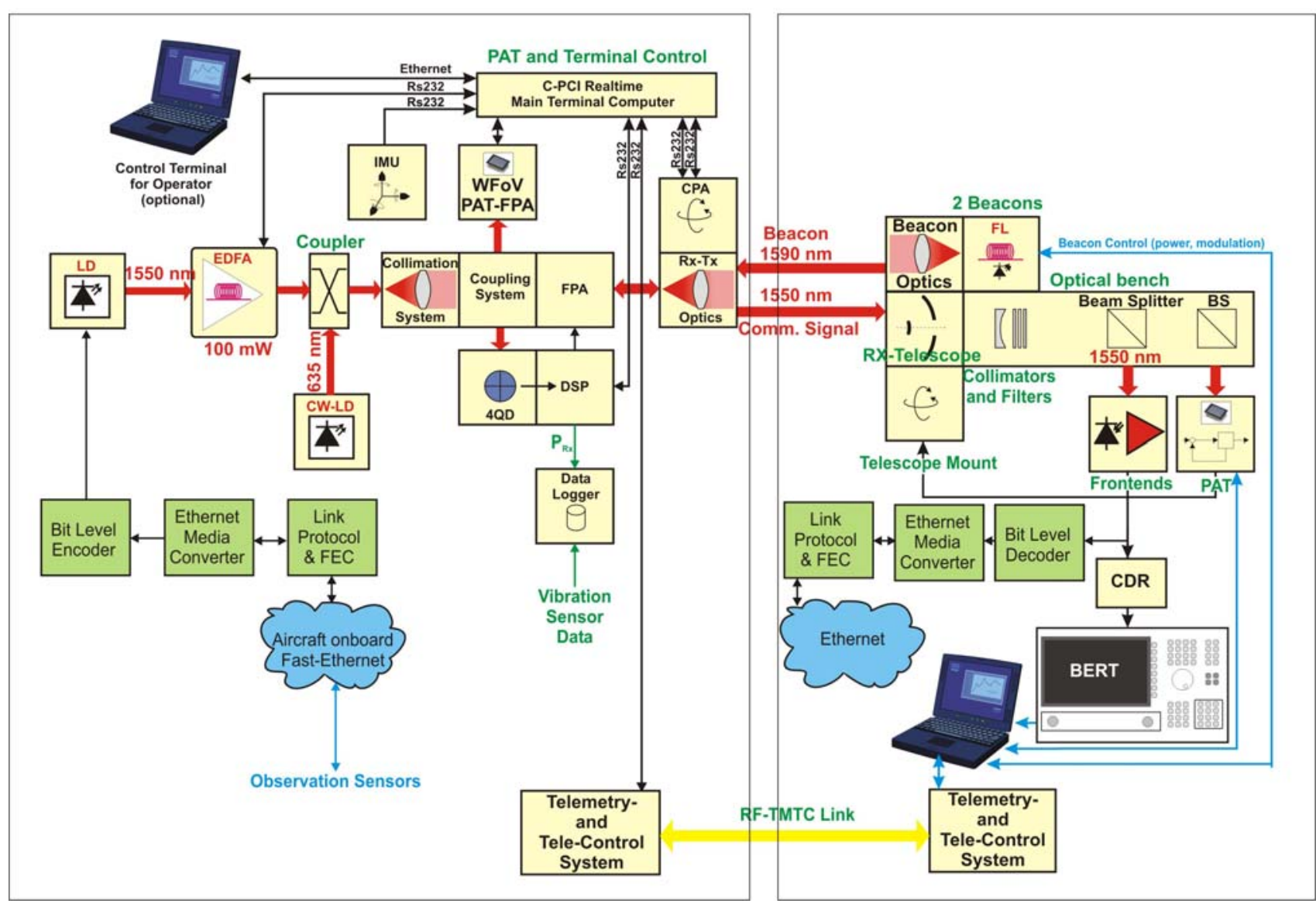

Figure 12: Schematic of the ARGOS system. In the left box the optical aircraft terminal setup can be seen and in the right the components of the OGS. 


\subsection{Summary of the different data downlink experiments}

Table 2 provides an overview of technical parameters of the different downlink trials (FT: Flight Terminal).

\begin{tabular}{|c|c|c|c|c|c|}
\hline & $\begin{array}{l}\text { KIODO } \\
(2006)\end{array}$ & $\begin{array}{l}\text { LCTSX-dlOP } \\
(2008 / 09)\end{array}$ & $\begin{array}{l}\text { CAPANINA } \\
\text { (2005) }\end{array}$ & $\begin{array}{l}\text { ARGOS-FastE } \\
(2008 / 09)\end{array}$ & $\begin{array}{c}\text { ARGOS-GbE } \\
\text { (2010) }\end{array}$ \\
\hline Type of Link & LEO-dl & LEO-dl & HAP-dl & $\begin{array}{l}\text { Aircraft-dl at } \\
\text { mid-altitude }\end{array}$ & $\begin{array}{l}\text { Aircraft-dl at } \\
\text { mid-altitude }\end{array}$ \\
\hline Channel Data Rate & $50 \mathrm{Mbps}$ & $5.6 \mathrm{Gbps}$ & $\begin{array}{l}\text { 622Mbps and } \\
1.25 \mathrm{Gbps}\end{array}$ & $\begin{array}{l}125 \mathrm{Mbps} \text { and } \\
155 \mathrm{Mbps}\end{array}$ & $1.25 \mathrm{Gbps}$ \\
\hline FT Altitude & $610 \mathrm{~km}$ & $514 \mathrm{~km}$ & $22 \mathrm{~km}$ & $3 \mathrm{~km}$ & $3 \mathrm{~km}$ \\
\hline Distance & $\begin{array}{c}2540 . .840 \mathrm{~km} \\
\left(3^{\circ} \text { to } 44^{\circ} \text { el.) }\right.\end{array}$ & $1728 \ldots 514 \mathrm{~km}$ & 64km (max.) & $10 . .85 \mathrm{~km}$ & $10 . .100 \mathrm{~km}$ \\
\hline $\begin{array}{l}\text { Min. Elevation with } \\
\text { tracking-lock }\end{array}$ & $3^{\circ}$ & $\sim 10^{\circ}$ & $\sim 18^{\circ}$ & $\sim 2,5^{\circ}$ & $\sim 1,7^{\circ}$ \\
\hline Modulation Format & $\mathrm{IM} / \mathrm{DD}$ & homodyne BPSK & $\mathrm{IM} / \mathrm{DD}$ & $\mathrm{IM} / \mathrm{DD}$ & $\mathrm{IM} / \mathrm{DD}$ \\
\hline FT Tx-power & $100 \mathrm{~mW}$ & $0.7 \mathrm{~W}$ & $100 \mathrm{~mW}$ & $100 \mathrm{~mW}$ & $1 \mathrm{~W}$ \\
\hline $\begin{array}{l}\text { Wavelength } \\
\text { downlink }\end{array}$ & $847 \mathrm{~nm}$ & $1064 \mathrm{~nm}$ & $1550 \mathrm{~nm}$ & $1550 \mathrm{~nm}$ & $1550 \mathrm{~nm}$ \\
\hline $\begin{array}{l}\text { max. Angular rate at } \\
\text { Ground Station }\end{array}$ & $<1 \%$ s & $<1 \%$ s & $<0.1 \% \mathrm{~s}$ & $\sim 2 \% \mathrm{~s}$ & $\sim 2 \% \mathrm{~s}$ \\
\hline $\begin{array}{l}\text { Downlink signal } \\
\text { divergence FWHM }\end{array}$ & $5.5 \mu \mathrm{rad}$ & $<10 \mu \mathrm{rad}$ & $1.25 \mathrm{mrad}$ & $2.2 \mathrm{mrad}$ & $\sim 500 \mu \mathrm{rad}$ \\
\hline $\begin{array}{l}\text { FT effective Tx- } \\
\text { aperture }\end{array}$ & $260 \mathrm{~mm}$ & $135 \mathrm{~mm}$ & $3 \mathrm{~mm}$ & $3 \mathrm{~mm}$ & $5 \mathrm{~mm}$ \\
\hline FT Tracking aperture & $260 \mathrm{~mm}$ & $135 \mathrm{~mm}$ & $25 \mathrm{~mm}$ & $30 \mathrm{~mm}$ & $30 \mathrm{~mm}$ \\
\hline $\begin{array}{l}\text { FT Pointing } \\
\text { Mechanism }\end{array}$ & CPA and FPA & CPA and FPA & CPA only & CPA only & $\mathrm{CPA}$ and FPA \\
\hline OGS Uplink Beacon & $808 \mathrm{~nm}$ & $1064 \mathrm{~nm}$ & $808 \mathrm{~nm}$ & $1590 \mathrm{~nm}$ & $1590 \mathrm{~nm}$ \\
\hline $\begin{array}{l}\text { OGS Uplink Beacon } \\
\text { Divergence }\end{array}$ & $5 \mathrm{mrad}$ & $2 \mathrm{mrad}$ & $5 \mathrm{mrad} \& 10 \mathrm{mrad}$ & $5 \mathrm{mrad}$ & $5 \mathrm{mrad}$ \\
\hline $\begin{array}{l}\text { OGS Uplink Beacon } \\
\text { Power }\end{array}$ & $\max .2 * 8 \mathrm{~W}$ & $\max .2 * 4,5 \mathrm{~W}$ & $\max .2 * 5 \mathrm{~W}$ & $\max .2 * 5 \mathrm{~W}$ & $\max .2 * 5 \mathrm{~W}$ \\
\hline $\begin{array}{l}\text { Sensitivity of data } \mathrm{Rx} \\
\text { / } \mathrm{BER}=10^{-6}\end{array}$ & $\sim 10 \mathrm{nW}$ & NA & 70nW (1.25Gbps) & $16 \mathrm{nW}(155 \mathrm{Mbps})$ & $<150 \mathrm{nW}$ \\
\hline $\begin{array}{l}\text { Typ. average Rx- } \\
\text { power on data- } \\
\text { detector during trials }\end{array}$ & $\begin{array}{l}20 \mathrm{nW} . .1 \mu \mathrm{W} \\
\text { (depending on } \\
\text { elevation) }\end{array}$ & NA & $1 \mu \mathrm{W}$ & $100 \mathrm{nW}$ & $1 \mu \mathrm{W}$ \\
\hline
\end{tabular}

Table 2: Parameters of the different data downlinks. All links were received by the $40 \mathrm{~cm}$ aperture of OGS-OP.

Note 1: Distance and thus minimum elevation in CAPANINA were limited by the flight trajectory dictated by local wind conditions. Typical values for operational HAP-downlinks would be up to $300 \mathrm{~km}$ distance and thus down to $2.5^{\circ}$ elevation. Also, an operational aerostatic HAP would be geostationary with angular rates close to zero.

Note 2: All stated measured values represent our experimental trial results, not absolute performance limits of the involved hardware. 


\section{SUMMARY AND OUTLOOK}

The different links are exposed to attitude disturbances of varying degree. While satellite platforms are very stable and can be controlled precisely, HAPs have to deal with the stratospheric wind impact. Low-altitude aircraft downlinks are heavily affected by turbulence, aircraft maneuvers, and engine-vibrations. Simplified, the lower the FT, the higher the remaining tracking error. On the other hand, to ensure illumination of the ground station with a certain required intensity, the divergence angle must be matched to the link distance. Fortunately, both requirements match with each other, meaning that the shorter links closer to the ground with the higher tracking error also allow broader beam divergence. As a rule of thumb with gaussian distributed remaining pointing error and when forward error correction coding (FEC) is applied for fading mitigation, the divergence angle FWHM should be at least four times $\sigma$ (PointingError). Figure 13 summarizes this relation: The blue solid line shows the required FWHM-divergence angle to achieve the required signal strength at the OGS to perform the link (based on individual link budget calculations). The red dotted line gives the actual achieved trial values of $4^{*} \sigma$ (PointingError), while the green dashed line shows the divergence angles of the different terminals. For the A/C-downlink the measured values of the first test flights in 2008 without FPA (FinePointing Assembly) are used, for LEO the OICETS-values, and for GEO the values of the SILEX-terminals (Semiconductor Inter-Satellite Link Experiment, by ESA) are used as reference.

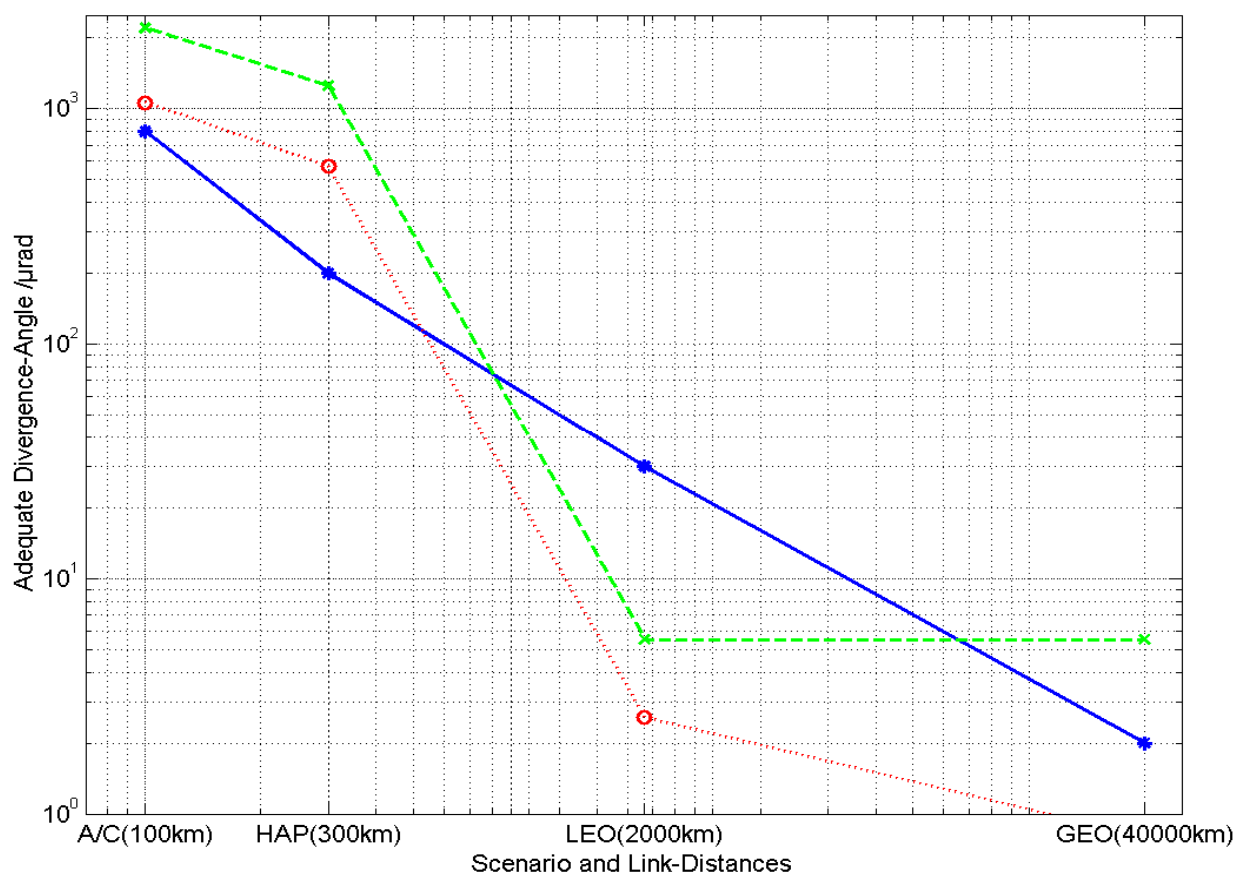

Figure 13: Relation between Link distance, remaining pointing error (dotted red), actual FWHM divergence angle (dashed green), and required FWHM beam divergence (solid blue). A typical set of parameters is used for link budget calculations here: $1.25 \mathrm{Gbps}, 100 \mathrm{~mW}$ Tx-power, $0.4 \mathrm{~m} \mathrm{Rx}$-aperture diameter, and receiver sensitivity is $70 \mathrm{nW}$. See text above for explanations.

Obviously, the currently achieved values of A/C and HAP downlinks are still too large for the 1.25Gbps link (red line is above the solid blue reference line). Smaller Tx-divergence together with a fast Fine-Pointing Assembly (four quadrant tracker and tip-tilt mirror) is required here. The LEO-terminals perform better than necessary, which is due to the fact that they were designed for inter-satellite links with longer distances and smaller Rx-apertures. The GEO-downlink of SILEX was designed for a much lower datarate $(2 \mathrm{Mbps})$ which explains why its Tx-divergence would be too large for transmitting $1.25 \mathrm{Gbps}$ to the small Rx-aperture of OGS-OP.

Regarding coherent transmission formats for atmospheric links, either AO technologies or coherent array receiver concepts need to be applied for enabling coherent transmission through atmospheric IRT with large receiver apertures, 
i.e. in long-range downlinks from GEO or exploration missions. Direct detection receiver concepts have shown to be robust in link scenarios that have lower receiver sensitivity constraints, i.e. shorter link range. The $15 x x n m$ wavelength features better eye-safety (as it is crucial in near-ground applications) as well as less disturbing solar background light compared to 8xxnm technology. Also in terms of available output power from EDFAs together with possible modulation bandwidth, 15xxnm with InGaAs-APD receivers is more future-proof than $8 \mathrm{xxnm}$.

High-speed error detection/correction is an enabling technology in any link case that suffers from IRT. Proactive error recovery mechanisms (e.g. forward error correction, FEC), possibly combined with reactive techniques (e.g. automatic repeat request protocols, ARQ), are crucial for securing the data transmission in IRT fading channels. Today, packetbased link layer approaches are available that work close to the Singleton-bound. For example, FEC-schemes exist which can recover packet-erasures up to $5 \%$ below their redundancy [9]. I.e. a code that causes $40 \%$ FEC-redundancy can recover all the data lost during fades if the total amount of lost data does not exceed $35 \%$. FEC must be matched to the specific transmission channel (IM/DD or coherent; shot- or thermal-noise limited), as these show different fading behavior. In ARGOS we will apply proactive error recovery based on packet layer FEC with Ethernet-packets in future.

\section{ACKNOWLEDGEMENTS}

The authors acknowledge the support of the German Space Agency, the European Space Agency (ESA), TesatSpacecom, and the German Space Operation Center (GSOC) during the LCT-downlinks from TerraSAR-X. We appreciate the cooperation and kind support of Japan's JAXA in KIODO. We also want to thank our colleagues from DLR's flight operations department at Oberpfaffenhofen for their support during ARGOS flight tests and all colleagues of DLR's Optical Communications Group.

\section{ABBREVIATIONS}

$\begin{array}{ll}\text { AO } & \text { Adaptive Optics } \\ \text { ARGOS } & \text { Airborne wide area high altitude monitoring system } \\ \text { dl } & \text { downlink } \\ \text { EDFA } & \text { Erbium Doped Fiber Amplifier } \\ \text { EO } & \text { Earth Observation } \\ \text { FoR } & \text { Field-of-Regard (e.g. of EO-sensor) } \\ \text { FT } & \text { Flight Terminal } \\ \text { GEO } & \text { Geostationary Earth Orbit } \\ \text { HAP } & \text { High Altitude Platform } \\ \text { IRT } & \text { Index-of-Refraction Turbulence } \\ \text { JAXA } & \text { Japan Aerospace Exploration Agency } \\ \text { KIODO } & \text { Kirari Optical Downlink to Oberpfaffenhofen, } \\ \text { Kirari } & \text { second name of OICETS } \\ \text { LCTSX } & \text { Laser Communications Terminal onboard TerraSAR-X } \\ \text { LEO } & \text { Low Earth Orbit } \\ \text { NICT } & \text { National Institute of Information and Communications Technology, Japan } \\ \text { OGS-OP } & \text { Optical Ground Station Oberpfaffenhofen } \\ \text { OICETS } & \text { Optical Inter satellite Communications Engineering and Test Satellite, by JAXA } \\ \text { SAR } & \text { Synthetic Aperture Radar } \\ \text { TerraSAR-X } & \text { German EO-Satellite with Synthetic Aperture Radar in X-Band }\end{array}$




\section{REFERENCES}

[1] Jono, T., Takayama, Y., Perlot, N., et al: "Report on DLR-JAXA Joint Experiment: The Kirari Optical Downlink to Oberpfaffenhofen (KIODO)", JAXA, ISSN 1349-1121, 2007

[2] Horwath, J., Perlot, N., Knapek., M, Moll, F.: "Experimental verification of optical backhaul links for high-altitude platform networks: Atmospheric turbulence and downlink availability", International Journal of Satellite Communications and Networking, 25(5), 501-528, 2007

[3] Horwath, J., Fuchs., C.: "Aircraft to Ground Unidirectional Laser-Comm. Terminal for High Resolution Sensors", Proc. of SPIE 7199, 2009

[4] Giggenbach, D. Epple, B., Horwath, J., Moll, F.: "Optical Satellite Downlinks to Optical Ground Stations and HighAltitude Platforms", in Advances in Mobile and Wireless Communications: Views of the 16th IST Mobile and Wireless Communication Summit, Springer, 2008

[5] Barbiera, C., Delauréb, B., Laviec, A.: "Strategic Research Agenda for High-Altitude Aircraft and Airship Remote Sensing Applications", USE-HAAS workshop, Antwerpen, October 2006

[6] Knapek, M., Horwath, J., Perlot, N., Wilkerson, B.: "The DLR Ground Station in the Optical Payload Experiment (STROPEX) - Results of the Atmospheric Measurement Instruments", Proc. of SPIE 6304, 2006

[7] Smutny, B., et al.: "In-orbit verification of optical inter-satellite communication links based on homodyne BPSK", Proc. of SPIE 6877, 2008

[8] Perlot, N.: "Turbulence-induced fading probability in coherent optical communication through the atmosphere", Applied Optics, 46 (29), p. 7218 - 7226, 2007

[9] Henniger, H., Gonzalez, A.: "Transmission Scheme and Error Protection for Simplex Long-Distance Atmospheric FSO Systems", Special issue of the Mediterranean Journal of Electronics and Communications on Hybrid RF and Optical Wireless Communications, 2006 